\title{
Research on A Special Scarifier Mechanism With Finite Element Analysis Method
}

\author{
Jiandong Jiang ${ }^{\mathrm{a}}$, Willem Hoogmoed ${ }^{\mathrm{b}}$, Guoxing Tao ${ }^{\mathrm{a}}$, Jie Gao ${ }^{\mathrm{a}}$, Xian Zhang ${ }^{\mathrm{a}}$ \\ ${ }^{a}$ College of Mechanical Engineering, ZheJiang University of technology, Hangzhou, P.R.China \\ ${ }^{\mathrm{b}}$ Farm Technology Group, Wageningen University, Wangeningen, The Netherlands \\ Correspond author: Xian Zhang, email:jiangjd@zjut.edu.cn
}

\begin{abstract}
A scarifier mechanism with rotary tillage and antirotary grubbing is proposed for inducing the power of tillage in hardens soil. MAT147 material modal is amended by experimental method and soil high-speed cutting finite element modal is build through SPH method, further, the tools parameter of proposed mechanism and soil cutting speed are studied by FEA numerical simulation through orthogonal experiments method. Finally, the result shows that the proposed mechanism with proper structural parameters and work speeds can reduce the requirement of power of tillage and increase the working efficiency of small agricultural machinery.
\end{abstract}

Keywords-hardens soil, scarifier; 3D numerical simulation; small agricultural machinery.

\section{INTRODUCTION}

Because of the limitation of original soil conditions ,or the labor mobility leading lax management of cash crops for, or the over-reliance on chemical fertilizers making low soil organic matter content and low water content, the soil in Southeast China mountainous hilly country is getting terrible hardens soil presently. The agricultural development of mountainous hilly country is seriously affected. Therefore, it is an urgent need to research and develop a agricultural machinery with mall cutting torque, deeply-loosening tillage and stable manipulation, and applying anti-rotary grubbing hardens soil technology in agricultural engineering is one of the core technologies in small power deeply-loosening tillage operation, but the research in traditional agricultural machinery design methods and soil mechanical cultivation does not meet modern agricultural machinery needs of the digital design and optimization. Thus, the last two decades, experts and researchers in the world have done a lot of research work in soil tillage and machinery for the design of digital tools and research methods; tillage equipment of digital principles and digitization and optimization got flourishing development.

To save as much as possible the process of tillage power consumption and energy-saving agricultural machinery operations in the agricultural machinery, many scholars at home and abroad have done a lot research and practice in the rotary cutter rotation to the impact on farming and its energysaving effect over years. Study shows that: rotary tillage is suitable to plant a thin loose soil, and has powerful capability to shred stubble, weeds, green manure crop, furrow slice broken, fluctuations in farming, poor stability. anti-rotary grubbing tillage can cutting from the plow layer at the bottom of the soil to upward, as it begin cutting, the thickness of furrow slice is relatively thin, the force on cutter shaft is uniform and smooth, vertical force of its rotary reaction force from top to bottom which ensure a good farming Deep stability, roughness in gully bottom of the antirotary grubbing tillage is not hard ditch smaller, and because of reducing average thickness of cut furrow slice, it improves the quality of the broken stone

We are targeting the South Hills, especially the tea hardens soil, because of its low moisture content and hard soil, we propose to use the method that rotary and anti-rotary work at the same time to the deep loose soil compaction. To consider the rotation for deep plowing operation is difficult, that, due to the role of soil resistance, unit is easy to produce a strong vibration, while to consider for the mountainous foothills of the mostly small-scale operations equipment which is small size, light weight and small inferior adhesion, we use anti-rotary grubbing can make up for the shortness of the above. Therefore, using the above-mentioned method that rotary tillage and anti-rotary grubbing loose screwdrivers to complete compaction of soil deep plowing operations, its tool design and determination of operating parameters is very $t$ important. Using the traditional method of soil cell test will cost a lot of money and time, but through the three-dimensional numerical simulation of anti-rotary grubbing tool cutting soil process, and do some research in the structure, kinematic parameters and demands on the power of anti-rotary grubbing cutter shaft which is suitable to be used in harden soil deep plowing operations, as well as for studies to guide the development of anti-rotary grubbing machinery. At the same time, using three-dimensional numerical approximation of numerical simulation techniques to simulate the cutting tool on soil process research

\section{Principle OF ANTI-Rotary GRUBBING Machinery}

Using three-dimensional numerical simulation technique can reproduce the process of cutter shaft cutting the soil on a computer the process of the soil, while obtain the relevant data which will help to optimize the motion parameters of cutting shaft and machinery based on the base. However, the authenticity of numerical simulation result is related to the numerical simulation technology and the environmental conditions. This paper is based on the SPH method for numerical simulation technique and its environmental conditions, including determination of soil parameter, determination of machinery structure and motion parameters, as well as determination of cutting shape and so on. 


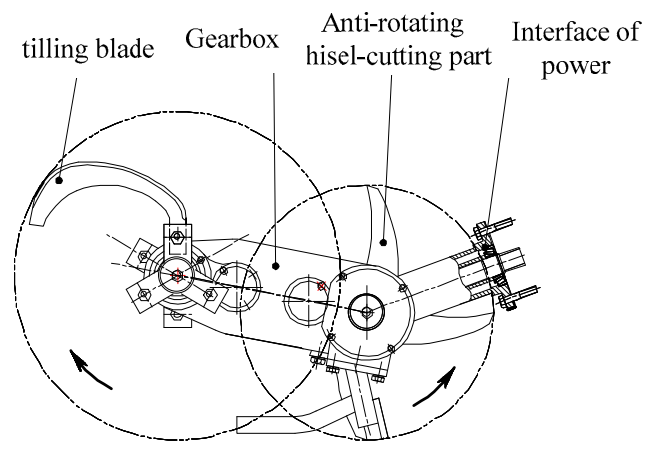

Figure 1. Scarifier mechanism of harden soil

\section{A. Kinetics of scarifier mechanism}

As Figure 1 shows that small agricultural machinery combines the advantage of rotary tillage and anti-rotary grubbing operations, using the anti-rotary grubbing at first ,then using rotary tillage overall layout. While working, the first anti-rotating chisel-cut ditch, so that a positive spin with a smaller lateral rotary bound state, to reduce the cutting torque and vibration amplitude. And, it is generated by rotating rotary operation unit forward thrust, you can offset some or all of the anti screwdrivers cutting resistance generated when the unit advance will help to overcome the small agricultural machinery' $s$ weakness that adhesion and mating power is small.

Small agricultural machinery will transfer power through a fast power interface to the working mechanism, drill through the gear driven counter-rotating cutter, while tilling blade is driven through the gears spin. According to previous research results and experience, we set the motion parameters as follows: machinery moving speed is 0.2$0.6 \mathrm{~m} / \mathrm{s}$, cutting speed of blades is $160-200 \mathrm{r} / \mathrm{min}$, and cutting rotary radius is $180 \mathrm{~mm}$.

\section{B. Structural parameters of reverse-rotary grubbing}

Seen as figure 2, When for the chisel-cutting blade at any time the location of the endpoint coordinates; the equations of motion is:

$$
\left\{\begin{array}{c}
x=R \cos \omega t+v_{m} t \\
y=R \sin \omega t
\end{array}\right.
$$

In the equation, $x, y$ stands for any time the location coordinates of Chisel-cutting part's endpoint, $R$ stands for rotation radius of chisel-type cutter's endpoint (roller blade radius of gyration); $t$ is the cutting time; $\omega$ stands for the tool rotation angular velocity, $v_{m}$ stands for the speed of machinery Advance unit.

Equation (1) reflects that when the cutting blade at work, the trajectory of its tip is a cycloid, as shown in Figure 2. The back of the tool and its tip to the rotation center and the angle between the connection structure of the gap angle, and I cycloid the tangent of the angle between the effective clearance angle; blades in front of the tip to the rotation and the connection between the cutting angle for the structure of soil angle; and I cycloid normal angle between the soil for the effective cutting angle.

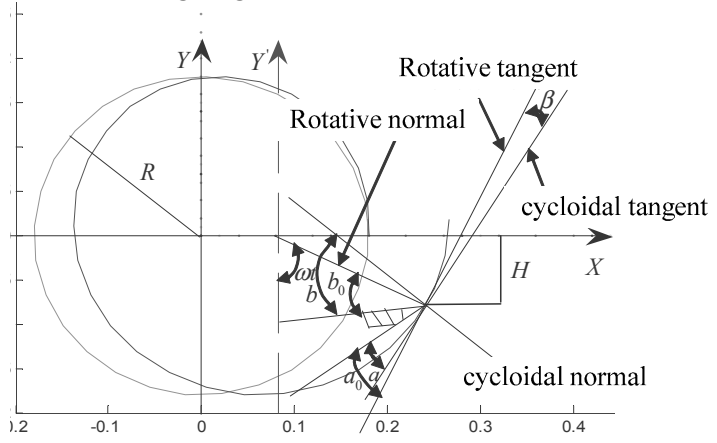

Figure 2. Kinetic parameters of anti-rotary chisel-cutting mechanism

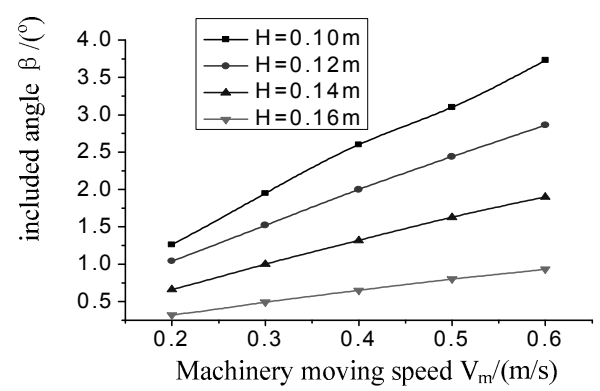

Figure 3. relation of included angle $\beta$ Vs moving speed with various

When $\omega t$ is moving in the cutting soil work area $\omega t=0 \sim \arccos (R-H / R)$, its effective clearance angle $a$ and the effective cutting angle $b$ is expressed separately by equation (3) and (4):

$$
\begin{gathered}
\beta=\arctan [-\cos (\omega t)]+\arctan \left(\frac{\frac{v_{m}}{\omega}-\sin \omega t}{\cos \omega t}\right)-\frac{\pi}{2} \\
a=a_{0}-\beta \\
b=b_{0}+\beta
\end{gathered}
$$

Where, $\beta$ is the included angle of that rotative tangent and the cycloidal tangent.

According to equation (2), we can get Figure 3. From Figure 3 , we can see: when the chisel cutter radius of gyration $R$ is $180 \mathrm{~mm}$, the rotational speed is $200 \mathrm{r} / \mathrm{min}$, farming depth is varied from $100 \mathrm{~mm}$ to $160 \mathrm{~mm}$, the range of $\beta$ is $0.32^{0} \sim-3.37^{0}$. According to the soil cutting dynamics, the effective clearance angle $a$ is greater than 0 to avoid the blade cutting back will not interfere with the soil, from the type 3 we can be seen that the structure after clearance angle should be larger than $3.37^{\circ}$. Considering tool's wearresistance, the cutting angle $a_{0}$ 's range should be $3^{0} \sim 10^{0}$. Soil cutting tool angle is the main angle affected soil shear chisel blade, based on experience, the range of structure soil cutting angle $b_{0}$ should be $60^{\circ} \sim 70^{\circ}$. H is the tillage depth.

To address the above machinery's working principle and structure parameters, we extract the relevant variables as in 
TABLE I. TECHNOLOGICAL PARAMETER OF NUMERICAL SIMULATION

\begin{tabular}{cc}
\hline volume weight of soil $\left(\mathrm{g} / \mathrm{cm}^{3}\right)$ & 1.57 \\
soil porosity $(\%)$ & 39.2 \\
machinery moving speed $(\mathrm{m} / \mathrm{s})$ & $0.2 \sim 0.5$ \\
Blade turning speed $(\mathrm{r} / \mathrm{min})$ & $2.67 \sim 3.33$ \\
radius of gyration of blade $(\mathrm{m})$ & 0.18 \\
the effective clearance angle $a_{0}\left({ }^{\circ}\right)$ & $3 \sim 10$ \\
structure soil cutting angle $b_{0}\left({ }^{\circ}\right)$ & $60 \sim 70$ \\
tillage depth $\mathrm{H}(\mathrm{m})$ & $0.09 \sim 0.12$ \\
Tillage width $(m)$ & $0.03 \sim 0.06$ \\
distribution density of blade $\left(\mathrm{g} / \mathrm{cm}^{3}\right)$ & 7.83 \\
Material Poisson ratio $(\sigma)$ & 0.38 \\
Material modulus of elasticity & 210 \\
$(G P a)$ & \\
\hline
\end{tabular}

Table 1 to take the numerical simulation of the compaction of soil inversion cutting depth of cut pine.

\section{ANTI-ROTARY GRUBBING MODEL AND ITS NUMERICAL SIMULATION WITH SPH METHOD}

A red soil sampled form tea garden of LONGJIN village, Hangzhou city of china is test by soil mechanics experiment system, its mechanics parameters are: volume weight of soil is $1.57 \mathrm{~g} / \mathrm{cm}^{3}$, porosity is $33.2 \%$, poisson's ratio is 0.38 , shear strength is $35 \mathrm{kpa}$, shear modulus is $31.05 \mathrm{Mpa}$, internal friction angle is $23.6^{\circ}$.

As the soil has a multi-phase, loose dispersion characteristics and properties, using a continuous physical theory and its numerical solution (finite element method or boundary element method) is difficult to describe the antirotation grubbing harden soil process [6]. Smooth Particle Hydrodynamics method (Smooth Particle Hydrodynamics, called SPH method) can effectively solve the problem.

The core of SPH method is interpolation theory [7]. When calculating the spatial derivative, we through a group called "nuclear function" points to "nuclear function evaluation" approximation [8], the continuity of the medium partial differential equations into integral form. The whole object is separated into a series of "particles", all physical quantities (density, pressure, velocity, internal energy, etc.) carried by these particles. In the calculation only involves discrete particles information, so points must be the sum of the neighboring particles.

In the three-dimensional numerical simulation study of cutting the soil, we the use MAT147 material model (it will be provided by * MAT_FHWA_SOIL card in LS-DYNA971) as the soil model. The model is based on the work of Abbo and Sloan and criteria a number of amendments to the MohrCoulomb [10], mainly to extend the moisture content, stress softening, kinematic hardening, deformation rate effects and cell deletion. The yield surface of revised criteria and the Mohr-Coulomb yield to appear too are hyperbolic fit, expressed as:

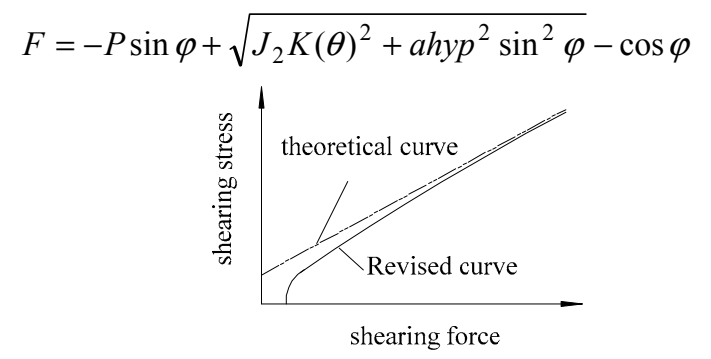

Figure 4. Comparison of Mohr-Coulomb yield surfaces in shear stress

In the equation, $P$ stands for pressure, as the internal friction angle, for the partial stress tensor in the square root of the second invariant for the deviatory stress plane in the polar angle, for cohesion, is to define the revised yield surface and standard Mohr-Coulomb yield surface paste between heterozygosis parameters. When using the default parameters 0:00, resume on the style standard MohrCoulomb yield surface, which take a value close to zero (see Figure 4).

In considering the length of smooth, artificial viscosity and its boundary processing requirements, we established process model as shown in Figure5. In order to ensure the accuracy of numerical simulation, we should make some constraints of the model's related boundary. Figure 6 reflects the results and data analysis of numerical simulation.

\section{DESIGN AND DATA PROCESSING OF NUMERICAL SIMULATION EXPERIMENT}

The general idea of this simulation experiments is: first determining the shapes of cutter, while optimizing the structure of the tip; second using the optimized parameters of the cutter to determine the motion parameters. Therefore, we will choose the orthogonal experimental method using group sequential design 2 to do the numerical simulation experiments.

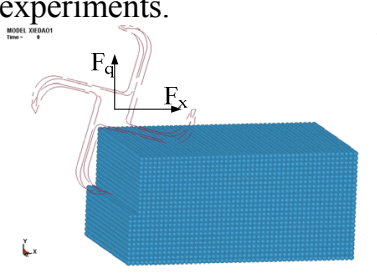

a) $3 \mathrm{D}$ modal

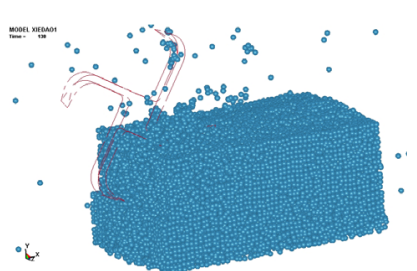

b) simulation of cutting process
Figure 5. 3D Model and Simulation of process
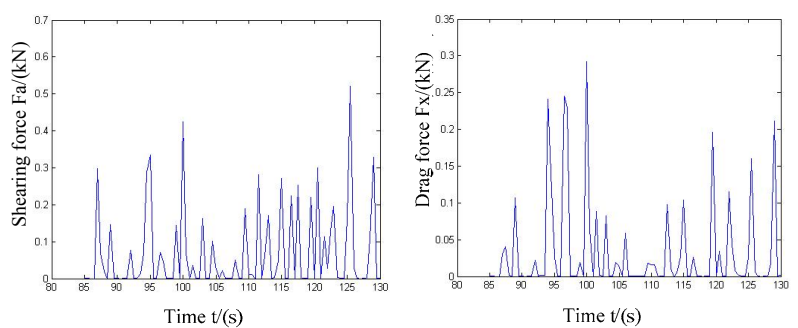

Figure 6. Data Graph of numerical simulation 
TABLE II.

OrThogonal Design OF CHISEL-CUTTING PARTS

\begin{tabular}{cccccc}
\hline Factors & \multicolumn{4}{c}{ Level } \\
& & 1 & 2 & 3 \\
\hline structure soil cutting angle $b_{0}\left({ }^{\circ}\right)$ & $\mathrm{A}$ & 60 & 65 & 70 \\
the effective clearance angle $a_{0}\left({ }^{\circ}\right)$ & $\mathrm{B}$ & 3 & & 6 & 10 \\
Blade shape & C & I & II & III \\
\hline
\end{tabular}

A. orthogonal experiments of structural parameters of cutting part

Chisel-type blade structure of clearance angle $a_{0}$, structure, soil cutting angle $b_{0}$ and the tool shape of soil when the tool cutting force as well as the machine's power consumption has a greater influence, through the numerical simulation, you can optimize the tip structure, so that in the Soil compaction under the conditions of farming, machine tool by the force and minimal power consumption. Based on preliminary analysis, listing the tip structure of factors and levels, see Table II.

To focus on studying the soil-breaking cutter's tip structure and obtain the optimal parameters of tip structure, in carrying out orthogonal experiment, we should the first tool on the tool shape on force to conduct numerical simulation, combined with operational requirements, in determining the shape of the tool under the premise of to carry out the optimal combination of structural parameters of cutting tool research. Numerical simulation, farming width, tillage depth for the crew forward speed, tool rotation speed, the angle of oblique blade, And seek the optimal combination of structural parameters. Numerical simulation results are in Table 3.

In Table III, $\mathrm{F}_{\mathrm{q}}$ reflects in the process of carrying out soil cutting chisel, chisel cutter got the maximum tangential force, the size of the force, reflecting the stable nature of the cutting process; $F_{x}$ is the largest forward resistance in the process of digging produced, the size of the force, reflecting the antispin operations generated by the size of the parasitic power; $\mathrm{T}_{\mathrm{p}}$ is average cutting torque.

TABLE III. KINETIC RESULTS OF VARIOUS SIMULATION EXPERIMENTS

\begin{tabular}{cccclll}
\hline scenarios & $\mathbf{A}$ & $\mathbf{B}$ & $\mathbf{C}$ & $\mathbf{F}_{\mathbf{q}}(K N)$ & $\mathbf{F}_{\mathbf{x}}(K N)$ & $\mathbf{T}_{\mathbf{p}}(N \cdot M)$ \\
\hline 1 & 1 & 1 & 1 & 0.636 & 0.843 & 21.45 \\
2 & 1 & 1 & 2 & 0.726 & 0.647 & 13.20 \\
3 & 1 & 1 & 3 & 0.456 & 0.458 & 11.28 \\
4 & 1 & 2 & 1 & 0.641 & 0.460 & 7.01 \\
5 & 1 & 3 & 1 & 0.915 & 0.489 & 15.43 \\
6 & 2 & 1 & 1 & 0.486 & 0.396 & 12.48 \\
7 & 2 & 2 & 1 & 0.318 & 0.224 & 8.93 \\
8 & 2 & 3 & 1 & 0.400 & 0.315 & 11.08 \\
9 & 3 & 1 & 1 & 0.371 & 0.727 & 9.89 \\
10 & 3 & 2 & 1 & 0.260 & 0.173 & 6.93 \\
11 & 3 & 3 & 1 & 0.604 & 0.562 & 12.29 \\
12 & 1 & 1 & 3 & 0.523 & 0.441 & 18.30 \\
13 & 1 & 2 & 3 & 0.650 & 0.585 & 14.16 \\
14 & 1 & 3 & 3 & 0.475 & 0.397 & 11.94 \\
\hline
\end{tabular}

\begin{tabular}{lllllll}
\hline 15 & 2 & 1 & 3 & 0.418 & 0.567 & 11.78 \\
16 & 2 & 2 & 3 & 0.493 & 0.495 & 10.22 \\
17 & 2 & 3 & 3 & 0.327 & 0.577 & 14.50 \\
18 & 3 & 2 & 3 & 0.385 & 0.276 & 7.50 \\
\hline 19 & 3 & 3 & 3 & 0.419 & 0.304 & 9.82 \\
\hline
\end{tabular}

According to the experimental data in Table III, we can see whether it is rectangular cutter $(\mathrm{C}=1)$,or oblique-shaped cutter $(\mathrm{C}=3)$, soil structure, cutting the average torque angle change on the TP have a greater impact, when the structure of cutting angle from the change to soil, the average torque will be reduced about the same time, greater sensitivity of oblique blade; either straight or oblique blade, structural changes after the clearance angle on the maximum tangential force $\mathrm{Fq}$, the biggest advance resistance $F_{x}$ and average torque Tp both would have an impact on the average torque $\mathrm{T}_{\mathrm{p}}$ is more sensitive, too large or too small, are all of the mechanical parameters will change at the same time there is an optimum angle; angle oblique blade and blade to planting time, If the structural parameters of the same tools, all kinds of mechanical parameters or less, but considering the oblique blade with a sliding-cutting at planting time, the role of beneficial weeds removed, the actual production process, using oblique blade would be more beneficial to . According to the number of times the value of simulation results, the design of the anti-rotating cutting blade cutter will be used oblique form, structure of the left and right clearance angle, structure Hikitsuchi angle, etc.

\section{B. The impact that Chisel-cut chisel cutting speed on the impact of torque}

Chisel cutting speed the work of sub-Corner Unit-line speed and forward speed. For the compaction of soil, its cultivation would be far greater than the power required for the soil in general, for the small units as possible in order to adapt to mountainous foothills of the operation, the tool shape, tip structural parameters under certain preconditions, Peeling the required power to the minimum as the goal, to carry out blade peeling speed, the speed of advance units of the optimal combination is very necessary. This experiment as the research object to bevel blade, blade angle structural parameters are: soil structure, cutting angle 700; structure of clearance angle; angle for both agriculture and width of $0.03 \mathrm{~m}$, till deep $0.12 \mathrm{~m}$, with the current small-scale operations machine operating parameters, the factors and levels in Table IV.

Orthogonal array to study the impact factors of each level, pairs of orthogonal experimental results corresponding to the processing and analysis, specifically in table V. That is, the above-mentioned orthogonal cutting simulation of counterrotating cutting equipment suffered the results of force and torque resistance. Can use the results of the analysis related to optimized design parameters of the unit.

TABLE IV. ORTHOGONAL DESIGN OF WORKING SPEEDS

\begin{tabular}{l|lllll}
\hline \multirow{2}{*}{ Factor } & \multicolumn{5}{|c}{ Level } \\
\cline { 2 - 6 } & 1 & 2 & 3 & 4 & 5 \\
\hline
\end{tabular}




\begin{tabular}{|c|c|c|c|c|c|}
\hline \multicolumn{3}{|c|}{$\begin{array}{l}\text { Machinery moving } \\
\text { speed }(\mathrm{m} / \mathrm{s})\end{array}$} & 0.2 & 0.3 & 0.6 \\
\hline \multicolumn{3}{|c|}{$\begin{array}{c}\text { Blade angular speed } \\
(\mathrm{r} / \mathrm{s})\end{array}$} & $\begin{array}{ll}\text { B } & 2.67\end{array}$ & 2.83 & 3.33 \\
\hline \multicolumn{2}{|c|}{ TABLE V. } & NEM & $\begin{array}{l}\text { IS RESULT } \\
\text { CENARIOS }\end{array}$ & VARIOUS SII & LATION \\
\hline scenarios & $A$ & B & $F_{q}(k N)$ & $F_{x}(k N)$ & $T(\mathrm{Nm})$ \\
\hline 1 & 1 & 1 & I & I & 7.79 \\
\hline 2 & 1 & 2 & / & I & 8.37 \\
\hline 3 & 1 & 3 & I & I & 8.59 \\
\hline 4 & 1 & 4 & 0.353 & 0.338 & 8.86 \\
\hline 5 & 1 & 5 & / & / & 8.97 \\
\hline 6 & 2 & 1 & 1 & I & 7.90 \\
\hline 7 & 3 & 1 & / & I & 10.11 \\
\hline 8 & 4 & 1 & / & I & 10.57 \\
\hline 9 & 5 & 1 & / & / & 13.88 \\
\hline 10 & 2 & 4 & 0.368 & 0.345 & I \\
\hline 11 & 3 & 4 & 0.457 & 0.374 & 1 \\
\hline 12 & 4 & 4 & 0.525 & 0.526 & 1 \\
\hline 13 & 5 & 4 & 0.582 & 0.555 & 1 \\
\hline
\end{tabular}

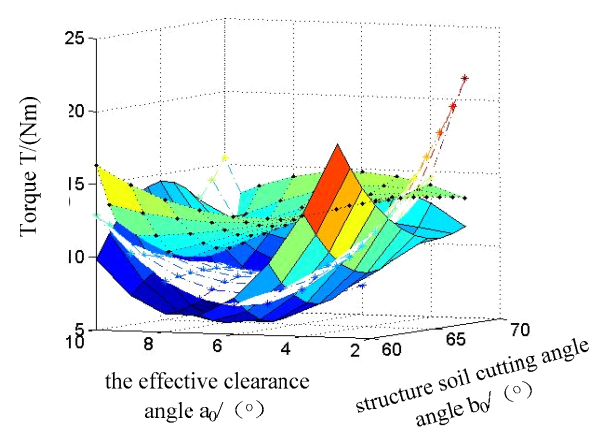

Figure 7. Torque variable of different numerical simulation

As can be seen from Table V: Anti-spin chisel cutting process, when the unit constant forward speed, tool rotation speed increases, the average distortion increased, but increased little; when the tool rotational speed constant, the increase in forward speed unit, will advance the tangential force and the resistance increase, while torque will quickly increase, but the rate of increase less than the forward speed. Its various parameters of power unit with the program, the situation is shown in Figure 7. It can be seen in the antiscrewdrivers rotary rotary-cut and positive combination of circumstances, to improve production efficiency, the appropriate unit to speed up forward speed, will be conducive to the improvement of energy efficiency.

\section{CONCLUSION}

In this paper, the Smoothed particle hydrodynamics $\mathrm{SPH}$ method in the compaction of soil cutting tools cutting process under the conditions of three-dimensional numerical simulation. Launched a counter-rotating pairs of chiselshaped cutting tool research; on the tip structure parameters were optimized; right use of the optimized anti-rotation chisel cutting tool of the motion parameters of the study, some qualitative conclusions drawn for the design and development for in a small job machine compaction of soil on the farm machines and tools provide a valuable theoretical basis. However, how to further improve the accuracy of numerical simulation, so that a more realistic simulation results, but also need to be more in-depth research. Meanwhile, the research methods used in this paper has highly efficient, rapid, low-cost characteristics, can be extended to other agricultural practices and soil-related studies of the machinery.

\section{ACKNOWLEDGEMENTS}

We would like to acknowledge the Natural Science Foundation of China (Grant 50805131), National Key Project of Scientific and Technical Supporting Programs Funded by Ministry of Science \& Technology of China (Grant 2006BAD11A10-02) for their support of this work.

\section{REFERENCES}

[1] LiuYongqing, Sang zhengzhong. Mathematical Model of Submerged Reverse-Rotary Tiller and Parameters Optimization [J]. TRANSACTIONS OF THE CHINESE SOCIETY OF AGRICULTURAL ENGINEERING ,2000,16(4):88 91.

[2] Kong Lingde, Sang zhengzhong. Evaluation of Crushing Soil from Rotary Tillage [J]. TRANSACTIONS OF THE CHINESE SOCIETY OF AGRICULTURAL MACHINERY ,1997,32(3):31 32,54.

[3] Ding Junhong, Jin Xianlong,Guo Yizhi,et al.. Study on 3-D Numerical Simulation for Soil Cutting with Large Deformation [J]. TRANSACTIONS OF THE CHINESE SOCIETY OF AGRICULTURAL MACHINERY,2007,38(4) :118 121.

[4] Xia Xiaodong,Wu Chongyou,Zhang Ruilin,et al.. Research and Design for Deepening Typeof Clockwise-Rotating Rotatiller [J]. TRANSACTIONS OF THE CHINESE SOCIETY OF AGRICULTURAL ENGINEERING,1999,15(1) :69 72 .

[5] Li JinQi.rotary ditcher[M].Beijing:Chinese agricultural machinery press, 1984.135-136.

[6] Gao Jianmin, Zhou Peng,Zhang Bing, et al. Development and test of high speed soil-cutting simulateion system based smooth particle hydrodynamics[J]. TRANSACTIONS OF THE CHINESE SOCIETY OF AGRICULTURAL ENGINEERING,2007,23(8):20 26.

[7] Monaghan JJ. Shock Simulation by the Particle Method SPH[J]. JCP, 1983,52:374

[8] GingoldRA,MonaghanJJ. Smoothed particle hydrodynamics theory and application to non-spherical stars[J].Monthly Notices of the Royal Astronomical Society, 1977,18:20 26.

[9] Monaghan JJ. preprint,Dept.of Math. Monash University, Clayton, Victoria 1990, 3168.

[10] Lewis Brett A. Manual for LS-DYNA soil material model 147[R].Department of Transportation: Federal Highway Administration,U.S.A, 2004. 